\title{
Immunotherapy Response Assessment in Neuro-Oncology Criteria
}

National Cancer Institute

\section{Source}

National Cancer Institute. Immunotherapy Response Assessment in Neuro-Oncology

Criteria. NCI Thesaurus. Code C131131.

Response assessment criteria for the evaluation of patients undergoing immunotherapy for the treatment of malignant glioma. These recommendations integrate guidance for the determination and confirmation of tumor progression based on the response assessment in neuro-oncology (RANO) working group and the immune-related response criteria (irRC). 